\title{
Portacosa, a new genus for the south-east Australian Grey Wolf Spider (Araneae, Lycosidae, Lycosinae)
}

\author{
Volker W. Framenau $1,2,3,4$ \\ 1 Phoenix Environmental Sciences Pty Ltd, 1/511 Wanneroo Road, Balcatta, Western Australia 6021, Australia. \\ 2 School of Biological Sciences, University of Western Australia, Crawley 6009, Western Australia, Australia \\ 3 Department of Terrestrial Zoology, Western Australia Museum, Locked Bag 49, Welshpool DC, Western Australia 6986, Australia \\ 4 Centrum für Naturkunde (CeNak), University of Hamburg, Martin-Luther-King-Platz 3, 20146 Hamburg, Germany
}

http://zoobank.org/AC4ED29D-692A-4F16-B300-58F7AE2BF008

Corresponding author: Volker W. Framenau (volker.framenau@phoenixenv.com.au)

Received 4 July 2017

Accepted 6 July 2017

Published 22 December 2017

Academic editor:

Martin Husemann

\section{Key Words}

taxonomy

systematics

Dingosa

trapdoor

\begin{abstract}
A new monotypic genus of Australian wolf spiders is proposed to accommodate the common south-east Australian Grey Wolf Spider, Portacosa cinerea gen. n. and sp. n. The genus includes large (total length 9.5-25.0 mm), uniformly grey-coloured wolf spiders with unique genital morphology, i.e. the ventral ridge of the tegular apophysis in the male pedipalp is comparatively sharp and situated towards its retrolateral edge, and the distinct anterior hood of the female epigyne is continuous with the inverted T-shaped median septum. Unlike most other members of the Lycosinae in Australia, the Grey Wolf Spider closes its burrow with a plug-like trapdoor. The species can be found in south-eastern mainland Australia (Queensland, New South Wales, Australian Capital Territory, Victoria, South Australia) and Tasmania. It prefers compacted, open and often sun-exposed habitats such as road verges and river banks, but can also be found in open woodlands and grasslands.
\end{abstract}

\section{Introduction}

When Hickman (1967) compiled his treatise on some common spiders of Tasmania, he associated Lycosa simsoni Simon, 1898 with what has since become known as the 'Grey Wolf Spider' (family Lycosidae Sundevall, 1833) (Fig. 1A-H). This is a common species in south-eastern mainland Australia and Tasmania that closes its burrow with a bath-plug like trapdoor (Fig. 1G-H). Following Hickman's publication the species was frequently referred to as Dingosa simsoni (Simon, 1898), as Roewer (1955a, b) had earlier based the genus Dingosa Roewer, 1955 on this species as type.

Hickman (1967) apparently did not examine the type material of L. simsoni, a female from Launceston and lodged in the Museum National d'Histoire Naturelle, Paris (MNHP4640; examined) (see Framenau and Baehr 2007). He probably relied on the superficial similarity of the epigyne of the Grey Wolf Spider with the illustration of D. simsoni by Roewer (1959; fig. 199). A recent revision of the genus Dingosa revealed, that $D$. simsoni is in fact not the Grey Wolf Spider, but a species of much different appearance and behaviour that builds a turret around the burrow entrance (Framenau and Baehr 2007). The misidentification by Hickman (1967) was facilitated by the abundance of the Grey Wolf Spider in suburban parks and gardens in Tasmania (see Other Material Examined below), whereas the true D. simsoni is uncommon in that state and only found around Launceston (Framenau and Baehr 2007).

The aim of this study is to establish a scientific name for the enigmatic and common Grey Wolf Spider and provide an up-to-date diagnosis and description to facilitate its identification. As the Grey Wolf Spider cannot be placed in any currently known wolf spider genus in Australia, a new genus is proposed to accommodate the species. 


\section{Material and methods}

This study is based on the examination of all major Australian museum and some overseas collections that resulted in a database of more than 20,000 records of Australian wolf spiders (more than 40,000 specimens examined). Descriptions are based on specimens preserved in $70 \% \mathrm{EtOH}$. A female epigyne was prepared for examination by submersion in $10 \%$ lactic acid for about one hour. Digital images were taken using a Leica DFC 295 digital camera attached to a Leica M205C stereo microscope. To increase depth of field, up to 20 images were merged using the software package Leica Application Suite 3.7.0. All measurements are in millimeters (mm). Morphological nomenclature follows Framenau and Baehr (2007) and Langlands and Framenau (2010).

Abbreviations: Eyes: anterior median (AME), anterior lateral (ALE), posterior median (PME), posterior lateral (PLE). Measurements (adult spiders, if not otherwise stated): total length (TL), carapace length (CL) and width $(\mathrm{CW})$, abdomen length (AL) and width (AW). Collections: Australian Museum, Sydney (AM); Museum Victoria, Melbourne (NMV); Queen Victoria Museum and Art Gallery, Launceston (QVMAG); Queensland Museum, Brisbane (QM); South Australian Museum, Adelaide (SAM); Tasmanian Museum and Art Gallery, Hobart (TMAG); Western Australian Museum, Perth (WAM).

\section{Taxonomy}

\section{Family Lycosidae Sundevall, 1833 \\ Subfamily Lycosinae Sundevall, 1833}

\section{Portacosa gen. n.}

http://zoobank.org/8CDB0C42-1A51-455F-B368-5BD6ED3C094B

Type species. Portacosa cinerea sp. n., designated here.

Diagnosis. Somatic morphology, in particular the lack of a distinct colour pattern on carapace and abdomen (in particular in live spiders, Fig. 1A-D), places Portacosa gen. n. in close affinity with Hoggicosa Roewer; however, males of Portacosa gen. n. lack the apical, dorsally bent setae on the cymbium tip and the median septum of the female epigyne is narrower anteriorly than the with of the posterior transverse part (see Langlands and Framenau 2010).

A putative apomorphy of the genus is the shape of the tegular apophysis. Unlike any other member of the family Lycosinae in Australia, the ridge of the tegular apophysis (connecting its apical point and ventral process; see Fig. $3 \mathrm{C}, \mathrm{D})$ is very sharp and situated more towards the retrolateral edge of the tegular apophysis, whereas it reaches from the apical point to a more central ventral process in other Lycosinae in Australia. This corresponds to a continuous edge of the anterior hood of the epigyne with the median septum (Fig. 4A, C), whilst this edge is often interrupted in other Lycosinae in Australia.

Portacosa gen. n. lacks any of the proposed synapomorphies of other Australian members of the Lycosinae, i.e. the tegular apophysis is not retrolaterally incised as in Venator Hogg, 1900 (Framenau 2015), the carapace lacks a Union-Jack pattern as in Tasmanicosa Roewer, 1959 (Framenau and Baehr 2016), the pedipalp lacks a large patch of apical setae as in Knoelle Framenau, 2006 (Framenau 2006a), the tegular apophysis is not elongated and spiders do not show turret-building behaviour as in Dingosa (Framenau and Baehr 2007), the carapace and abdomen are not dorsoventrally flattened as in Tapetosa Framenau, Main, Waldock \& Harvey, 2009 (Framenau et al. 2009), the tegular apophysis lacks apical serrations as in Costacosa Framenau \& Leung, 2013 (Framenau and Leung 2013), the abdomen is not dark with transverse light bands and spiders do not display turret-building behaviour as in Mainosa Framenau, 2006 (Framenau 2006b), and male pedipalp cymbia do not have a compound apical hook as in Venatrix Roewer, 1960 and Tuberculosa Framenau \& Yoo, 2006 (Framenau and Vink 2001; Framenau and Yoo 2006).

Etymology. The genus-group name is a composite noun derived from the Latin word portus - door, referring to the trapdoor-building behaviour of the type species and $-\cos a$, a generic ending used for genera in the family Lycosidae. The gender is feminine.

Description. Large wolf spiders (TL 10.5-25.0 mm). Males slightly smaller than females. Carapace longer than wide, dorsal profile straight in lateral view. Carapace colouration brown with indistinct darker radial pattern, covered by grey pubescence that is denser between eyes, in particular in males. Abdomen dorsally with indistinct median chevron-pattern, which is less distinct in females and covered with dense grey pubescence, ventrally uniformly yellow-brown. AME larger than ALE, row of AE slightly procurved and narrower than row of PME. Chelicerae with three promarginal teeth with the median largest and three large, equally-sized retromarginal teeth. Leg formula IV > I > II > III. Cymbium tip with approximately 20 straight spines (Fig. 3A, B). Tegulum of male pedipalp undivided (Fig, 3F); tegular apophysis with distinct ventral spur situated towards the retrolateral edge of the tegular apophysis, connected to apical point of tegular apophysis by sharp ridge (Fig. 3C, D). Embolus originating prolaterally on palea and curving ventrally around it, long and slender. Terminal apophysis broad and apically straight, pars pendula sickle-shaped (Figs 3E). Female epigyne with inverted T-shaped median septum, slightly longer than wide, anterior hoods distinct, connected continuously to medium septum (Figs 4A, C); spermathecal heads oval; spermathecal stalks S-shaped (Fig. 4B).

Composition. Portacosa gen. n. currently includes only a single species, $P$. cinerea gen. n. and sp. n.

Systematics. Portacosa gen. n. is a member of the subfamily Lycosinae Sundevall, 1833 based on the transverse orientation of the tegular apophysis that has a dorsal channel to guide the embolus (Dondale 1986). However, sistergroup relationships remain unclear. Portacosa cinerea gen. $n$. and sp. n. was included in a world-wide multigene molecular analysis of the Lycosidae as 'New Genus 6 

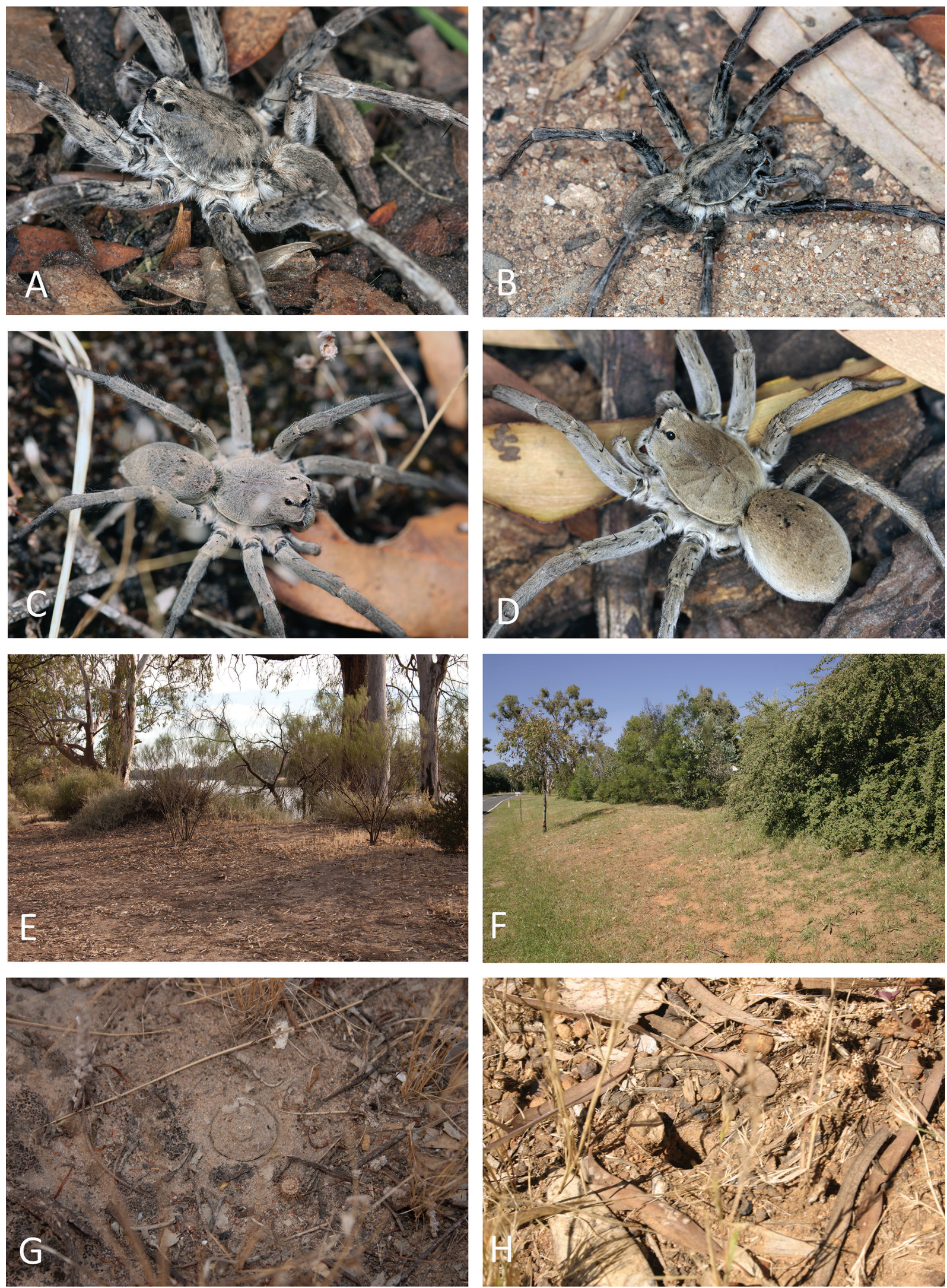

Figure 1. Live images and habitat of Portacosa cinerea gen. n. and sp. n. A, male holotype (WAM T68032) from near Apex Park, Mildura, Victoria. B, male, same locality. C, female (WAM T56062) from Specimen Hill Bushland Reserve, Bendigo, Victoria, D, female from Red Hill, Canberra, Australian Capital Territory (WAM T67906); E, open Eucalyptus camaldulensis riparian woodland, near Apex Park, Mildura, Victoria, where the holotype male (WAM T68032) was found. F, road embankment in Red Hill, Canberra, habitat of female WAM T67906. G, closed burrow of holotype male (WAM T68032). H, open burrow of female WAM T67906 from Red Hill, Canberra. 
sp.' (Murphy et al. 2006). Maximum parsimony analyses placed Portacosa gen. n. as sister to the South American Pavocosa gallopavo (Mello-Leitão, 1941), both of which combined represented a sister taxon to a clade including Hoggicosa, Hogna and Tasmanicosa. In contrast, Bayesian analyses placed Portacosa gen. n. in a poorly resolved polytomy including the above Australian genera as sister to Pavocosa Roewer, 1960. Similarly, a morphological phylogenetic analysis of largely Australian Lycosinae did not resolve sistergroup relationships of Portacosa gen. n. (included in the analysis as 'Grey Wolf Spider'); however, that analysis was mainly focused on resolving relationships within Hoggicosa (Langlands and Framenau 2010).

\section{Portacosa cinerea sp. $\mathbf{n}$.}

http://zoobank.org/8BB19DFB-9D74-477E-B57E-7DB3FDA309B0 Grey Wolf Spider

Figs 1-5

Lycosa simsoni Simon.- Hickman 1967: 80, figs 142, 143 (misidentification; see Framenau and Baehr 2007).

Material examined. Holotype male, Mildura, near Apex Park (3410'53'S, 14209'26'E, Victoria, AUSTRALIA), Framenau, V.W., Thomas, M.L., Ramamurthy, S., Eucalyptus camaldulensis riparian woodland, 11 January 2006 (WAM T68032). Paratype female, Mildura (341's, $142^{\circ} 09^{\prime} \mathrm{E}$, Victoria, AUSTRALIA), D. Mead-Hunter, 31 October 1984 (WAM T53550).

Other material examined (131 males, 120 females -8 with eggsacs, 3 with spiderlings - and 11 juveniles in a total of 214 records). AUSTRALIA: Australian Capital Territory: 1 female, Ainslie, 35 $16^{\prime}$ 'S, $149^{\circ} 9^{\prime} \mathrm{E}$ (ANIC); 1 female, Black Mountain, $35^{\circ} 16^{\prime} \mathrm{S}, 1^{\circ} 9^{\circ} 06^{\prime} \mathrm{E}$ (ANIC); 1

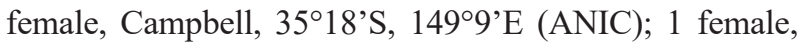
Red Hill, cr Hindmarsh Ave and Mugga Lane, 35²0'47'S, 14908'03'E (WAM T67906); 1 female, Yarralumla, $35^{\circ} 19^{\prime}$ S, $149^{\circ} 07^{\prime} \mathrm{E}$ (ANIC); New South Wales: 1 female, no data, no exact locality (AM KS86314); 2 females, Armidale, 30³1'S, $151^{\circ} 40^{\prime} \mathrm{E}$ (AM KS86364, KS86359); 2 females, Artarmon, $33^{\circ} 47^{\prime} \mathrm{S}, 151^{\circ} 11^{\prime} \mathrm{E}$ (AM KS86313, KS86324); 2 females, Ashfield, $33^{\circ} 53^{\prime} \mathrm{S}, 1^{\circ} 1^{\circ} 07^{\prime} \mathrm{E}$ (AM KS86278, KS86287); 1 female, 1 juv., Avalon, 333's,

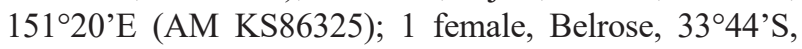

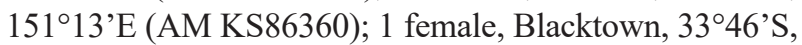
$150^{\circ} 55^{\prime} \mathrm{E}$ (AM KS86361); 1 female, Bombala, $36^{\circ} 55^{\prime} \mathrm{S}$, $149^{\circ} 15^{\prime} \mathrm{E}$ (AM KS86279); 1 female, Broken Hill, 31 ${ }^{\circ} 57^{\prime}$ 'S, $141^{\circ} 27^{\prime} \mathrm{E}$ (SAM NN14784); 1 male, same locality (WAM T62654); 1 female, Burwood, 335'S, $151^{\circ} 06^{\prime} \mathrm{E}$ (AM KS86277); 1 female, Callala Bay, $35^{\circ} 00^{\prime} \mathrm{S}, 150^{\circ} 43^{\prime} \mathrm{E}$ (AM KS53800); 1 male, Carlingford, $33^{\circ} 47^{\prime} \mathrm{S}, 151^{\circ} 03^{\prime} \mathrm{E}$ (AM KS4340); 1 female, Carlingford, 334ㅇ'S, $151^{\circ} 04^{\prime} \mathrm{E}$ (AM KS86282); 1 female, Carlton, 3358'S, $151^{\circ} 07^{\prime} \mathrm{E}$ (AM KS86281); 1 female, Chatswood, $33^{\circ} 48^{\prime} \mathrm{S}, 151^{\circ} 11^{\prime} \mathrm{E}$ (AM KS16519); 1 female, Coomba Station, West End, $28^{\circ} 30^{\prime} \mathrm{S}$, $150^{\circ} 28^{\prime} \mathrm{E}$ (NMV K8142); 1 male, Dunedoo, $32^{\circ} 00^{\prime} \mathrm{S}$, $149^{\circ} 23^{\prime} \mathrm{E}$ (AM KS82586); 1 female, Earlwood, $33^{\circ} 55^{\circ} \mathrm{S}$,
1510ㅇ'E (AM KS86319); 1 female, East Hills, 3357'S, $150^{\circ} 59^{\prime} \mathrm{E}$ (AM KS86280); 1 male, East Roseville, 334'ㄱ' $151^{\circ} 12^{\prime} \mathrm{E}$ (AM KS86363); 1 male, Eastwood, 334ㄱ'S, $151^{\circ} 05^{\prime} \mathrm{E}$ (AM KS21619); 1 male, Euglo State Forest, 33⒉'S, $147^{\circ} 15^{\prime}$ E (AM KS91371); 1 female, Euston Station, Lower Murray-Darling region, 34³1'49"S, 14247'9'E (AM KS67024); 2 males, Garston Station, Lower Murray Darling Region, 3347'37'S, 142 $00^{\circ}$ '14'E (AM, KS72695, KS91608); 1 male, 2.5 km NW Gin Gin, on road to Riverview Station, 31 ${ }^{\circ} 54^{\prime} 13$ 'S, $148^{\circ} 03^{\prime} 41^{\prime \prime} \mathrm{E}$ (AM KS76336); 1 male, Glenie Station, approx. $70 \mathrm{~km} \mathrm{~W}$ of Tottenham, 32॰19'45'S, 146 37'02'”E (AM KS76335); 1 male, Glenie Station, approx. $69 \mathrm{~km} \mathrm{~W}$ of Tottenham, 32¹7'35'S, 146³7'19'"E (AM KS70817); 1 female, Greenacre, $33^{\circ} 55^{\prime} \mathrm{S}, 151^{\circ} 03^{\prime} \mathrm{E}$ (AM KS86285); 1 male, Greenwich, 3349'S, $151^{\circ} 11^{\prime} \mathrm{E}$ (AM KS82600); 3 males, Gubatta, 333'03'S, 146³6'43”'E (QM S35935, S53765, S53869); 1 female, Gunnedah, 3059'S, 150 $15^{\circ} \mathrm{E}$ (AM

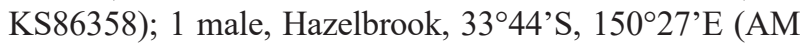

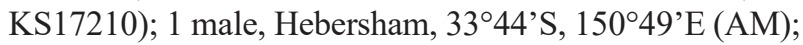
1 male, Homebush, $33^{\circ} 51^{\prime} \mathrm{S}, 151^{\circ} 05^{\prime} \mathrm{E}$ (AM KS8817); 1 female, Jenkins via Junee, 3452'S, 147³5'E (AM KS86288); 1 female, Kellyville, near Parramatta, 3342'S, 15057’E (AM KS86317); 1 male, Ki Downs Station, Lower Murray-Darling region, 3435'29'S, 142 34'33'E (AM KS67004); 1 male, Kinchega National Park, 32²9’S, $142^{\circ} 18^{\prime}$ E (AM KS69251); 1 female, Lane Cove, Sydney,

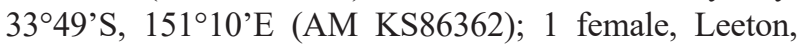
343'ㅇ, $146^{\circ} 24^{\prime} \mathrm{E}$ (AM KS86283); 1 male, 'Loxley` Station, approx. $34 \mathrm{~km} \mathrm{NW}$ of Tottenham, 32 $05^{\prime} 17^{\prime}$ 'S, $147^{\circ} 02^{\prime 2} 29^{\prime \prime} \mathrm{E}$ (AM KS70828); 7 males, Mallara Station, Lower Murray-Darling region, 3324'48'S, 142 $34^{\circ}$ '08' E (AM KS91593); 1 female, Murray River, S of Lake Victoria, 3358'S, $141^{\circ} 16^{\prime} \mathrm{E}$ (SAM NN16573); 1 female, Nar-

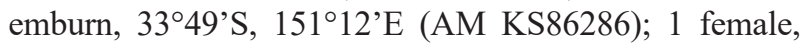
Narrandera, $34^{\circ} 45^{\prime} \mathrm{S}, 146^{\circ} 33^{\prime} \mathrm{E}$ (AM KS50421); 1 female,

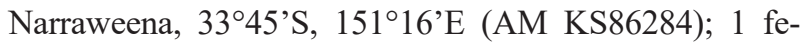

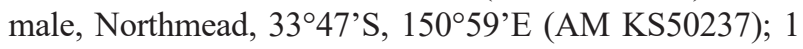

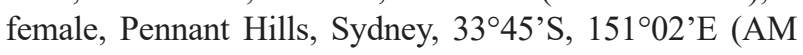
KS86316); 2 males, Popitah Station, Lower Murray-Darling region, 3309'16”S, 14141'44”E (AM KS92512); 3 males, Prill Park Station, Lower Murray-Darling region, 34³4'21'S, 142³9'05'E (AM KS67010); 1 male, Pulletop, 3401'49”S, $146^{\circ} 04^{\prime} 20^{\prime \prime} \mathrm{E}$ (QM S52831); 2 males, Pulletop, 33॰58'46”S, 14603'28'E (QM S53577); 1 male, 'Ronvue' Station, approx. $17 \mathrm{~km}$ SSE of Tottenham, 32²3'9'S, 147²6'40"E (AM KS70816); 1 female, Rose-

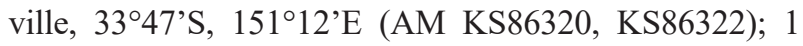
male, Round Hill Nature Reserve, 32॰59'20'S, 14605'06'"E (QM S52875); 1 male, Seven Hills, 3347'S, $150^{\circ} 56^{\prime} \mathrm{E}$ (AM KS54484); 1 female, same locality (AM

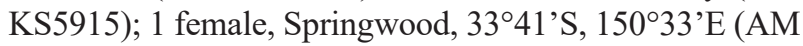

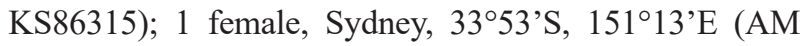
KS68881); 1 female, 2 juv., Tubrabucca, 31 ${ }^{\circ} 53^{\prime} \mathrm{S}$,

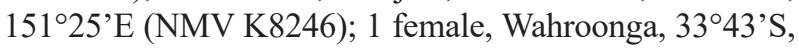
$151^{\circ} 08^{\prime} \mathrm{E}$ (AM KS86318); 1 female, Warraderry State Forest, Campsite, 3341'19'S, 148¹1'57'"E (AM KS85899); 3 males, Warrananga Station, Lower Murray-Darling Re- 

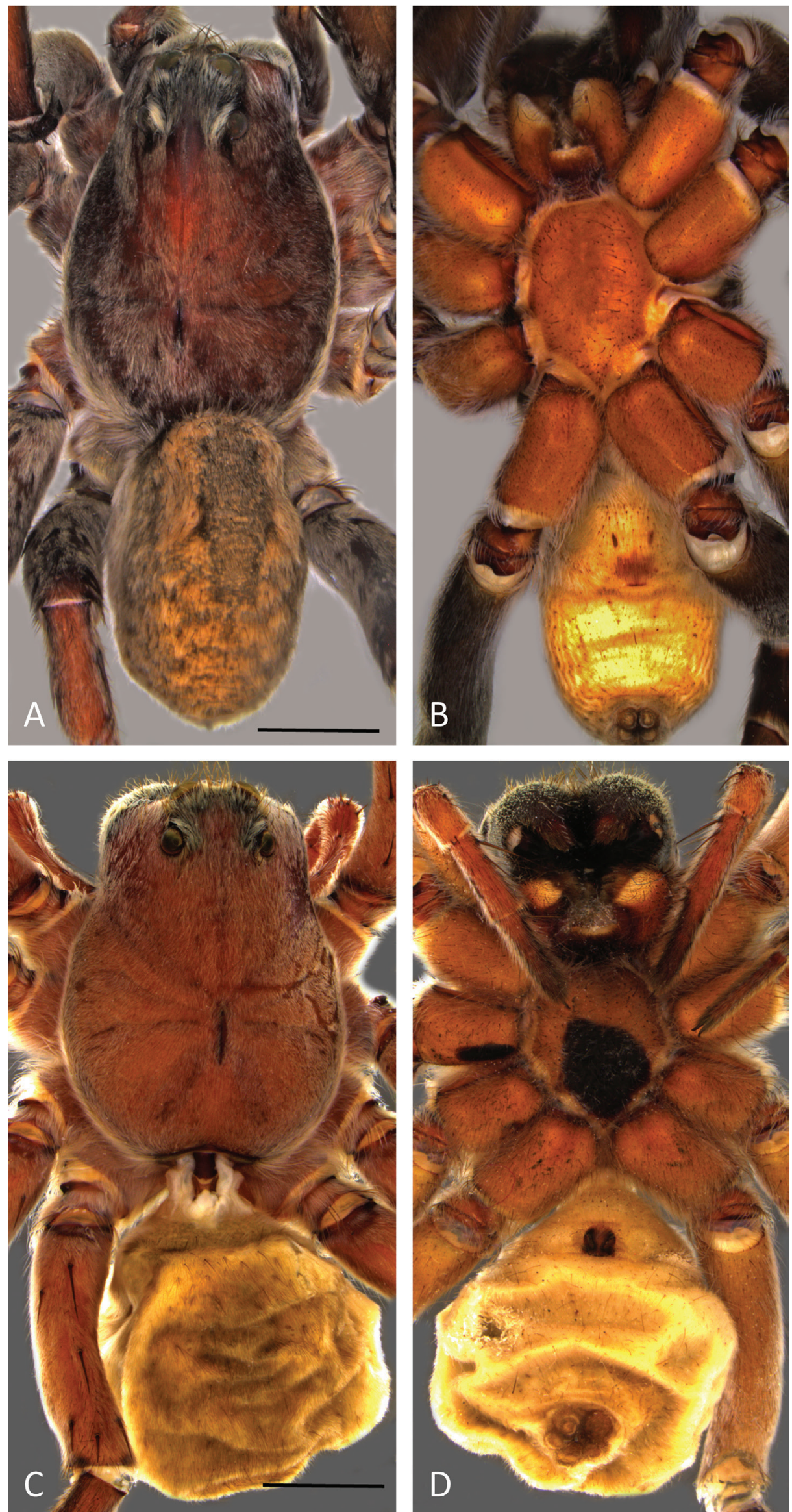

Figure 2. Portacosa cinerea gen. n. and sp. n., male holotype (WAM T68032) and female paratype (WAM T53550). A, B, male holotype in dorsal (A) and ventral (B) view. C, D, female paratype in dorsal (C) and ventral (D) view. Scale bar: A, B - 2.5; C, D - 3.1. 
gion, 3404'46"S, 141 39'21'"E (AM KS91590); 2 males, West Pymble, $33^{\circ} 45^{\prime} \mathrm{S}, 151^{\circ} 08^{\prime} \mathrm{E}$ (AM KS6150); 1 fe-

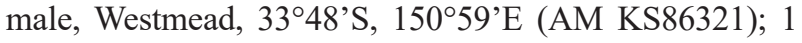
male, Yagoona, 33⒌'S, $151^{\circ} 01^{\prime} \mathrm{E}$ (AM KS82615); 4 males, Yara, 3256'48”'S, 146¹1'32”'E (AM KS79019); 1 male, same locality (AM KS79034); 2 males, same locality (AM); 5 males, Yara, 32॰51'54”S, 146¹1'21'"E (AM); 1 male, same locality (AM KS79033); 2 males, same locality (AM KS79037); 2 males, Yara, 32॰56’39”S, 14611'32'"E (AM KS85900); 4 males, same locality (AM KS79019); 1 male, Yathong Nature Reserve, 32 35'13”S, 14529'51'E (AM KS79021); 3 males, 1 juv., Yathong Nature Reserve, 3250'56”S, 146 $10^{\circ} 58^{\prime}$ 'E (AM KS79069). Queensland: 1 female, Birdsville Track to Mt Isa, no exact locality (NMV K8182); 1 female, 1 juv., Dalby, $27^{\circ} 11^{\prime} \mathrm{S}, 151^{\circ} 15^{\prime} \mathrm{E}$ (AM KS86323); 1 female, East Nanango, Alpaca Lodge, 264ㅇ's, $152^{\circ} 03^{\prime} \mathrm{E}$ (QM S61209); 1 male, Expedition Range National Park, 'Amphitheatre ' yards, $25^{\circ} 13^{\prime} \mathrm{S}, 1^{\circ} 9^{\circ} 01^{\prime} \mathrm{E}$ (QM S32469); 2 fe-

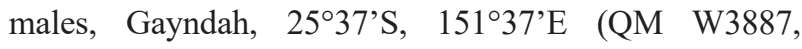
W3888); 2 females, 2 juv., Hurdle Gully, 14.8 km WSW Monto, 245' 'S, 15059'E (QM S44170); 1 female, Marlaybrook, Bunya Mountains, 26 $54^{\circ}$ 'S, 151³5'E (QM W5733); 1 female, Marlong Creek, Mt Moffatt Station, Koolalurra, 2501'S, $147^{\circ} 57^{\prime} \mathrm{E}$ (QM S70608); 1 female, Nanango, 26 $6^{\circ} 40^{\prime} \mathrm{S}, 1^{\circ} 2^{\circ} 00^{\prime} \mathrm{E}$ (QM S21661). South Australia: 1 male, Adelaide, Pitchford's, 345'' S, $138^{\circ} 36^{\prime} \mathrm{E}$ (WAM T64072); 1 male, Billiatt Conservation Park, 3459'29”'S, 140²8'22”'E (SAM NN16404); 1 male, 7 km W Coonalpyn, 3542'00'S, 13946'48'E (SAM NN16316); 2 males, 8 km SW Coonalpyn, 3544'42”S, 13946'44'E (SAM NN16317-8); 1 male, Cooper Creek, 2823'S, $137^{\circ} 41^{\prime} \mathrm{E}$ (SAM NN435); 1 male, Dog Lake

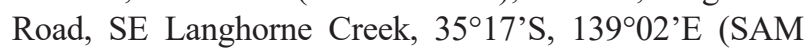
NN14772); 2 males, 1 female, same locality (SAM NN14773-5); 1 male, Goolwa, 35³0'S, 138 46'E (SAM NN14780); 3 males, Karte Conservation Park, 3507'03”S, 14043'33'E (SAM NN21354-6); 1 female, same locality (SAM NN21352); 1 male, same locality (SAM NN17088); 1 male, Katarapko Island, 34²4'16"S, 140³3'25'"E (ANIC); 1 female, Langhorne Creek, 35¹8'S, $139^{\circ} 02^{\prime} \mathrm{E}$ (SAM NN16415); 1 female, 8 km SE Langhorne Creek, $35^{\circ} 17^{\prime} \mathrm{S}, 1^{\circ} 9^{\circ} 02^{\prime} \mathrm{E}$ (SAM NN21357); 1 female, $22.5 \mathrm{~km}$ SW Loxton, $34^{\circ} 33^{\prime}$ S, $140^{\circ} 22^{\prime} \mathrm{E}$ (SAM NN14777); 1 male, Monarto Zoo, 3506'54'S, 13908'9'”E (SAM NN21654); 1 female, Nappyalla, $35^{\circ} 20^{\prime} \mathrm{S}, 1^{\circ} 9^{\circ} 07^{\prime} \mathrm{E}$ (SAM NN14768); 1 male, same locality (SAM NN14767); 1 female, same locality (SAM NN14764); 1 male, same locality (SAM NN14769); 1 female, same locality (SAM NN14765); 1 male, same locality (SAM NN14770); 1 male, same locality (SAM NN16414); 1 male, same locality (SAM NN14766); 1 male, 1 female, same locality (SAM NN14762-3); 1 male, 1 juv., 3 km NW Nappyalla, 35¹8'S, $139^{\circ} 05^{\prime} \mathrm{E}$ (SAM NN14771); 1 male, Naracoorte, 36 $57^{\circ} \mathrm{S}$, $140^{\circ} 45^{\prime} \mathrm{E}$ (SAM NN16479); 1 male, Ngarkat Conservation Park, Pertendi Hut Campsite, 35³8'17's, 14046'50"E (AM KS77362); 1 male, 8 km SE Peake, 3526'06”S, 139 58'55'E (SAM NN16315); 1 female,
Peebinga Conservation Park, 3459'16”S, 14048'12”E (SAM NN21358); Punkah Island, Chowilla, 335'42”S, 14057'01'"E (SAM NN679); 1 male, Rockleigh, 3457'S, $139^{\circ} 05^{\prime} \mathrm{E}$ (SAM NN14009); 1 male, Silas, W of Walpeup, $35^{\circ} 08^{\prime} \mathrm{S}, 142^{\circ} 02^{\prime} \mathrm{E}$ (SAM NN21353); 1 male, Stoneleigh Park, near Meningie, $35^{\circ} 32^{\prime}$ S, $1^{\circ} 9^{\circ} 30^{\prime} \mathrm{E}$ (SAM NN14782); 1 male, $9.9 \mathrm{~km}$ NE Woolumbool telephone exchange, 3643'04'S, 140²6'39'E (SAM NN16478). Tasmania: 1 female, no exact locality (QVMAG 13:44913); 1 male, 'Milford ', Cambridge, 41 ${ }^{\circ} 48^{\prime}$ 'S, $147^{\circ} 31^{\prime} \mathrm{E}$ (TMAG J1525); 1 male, Avoca, 41 46 'S, $147^{\circ} 43^{\prime} \mathrm{E}$ (TMAG J864); 1 female, 1 juv., Bellerive, Hobart, $42^{\circ} 52^{\prime} 51^{\prime \prime S}, 147^{\circ} 22^{\prime} 01^{\prime \prime} \mathrm{E}$ (WAM T55285); 1 male, Bridgenorth, $41^{\circ} 22^{\prime} \mathrm{S}, 146^{\circ} 58^{\prime} \mathrm{E}$ (QVMAG 13:39772); 2 females, Bridgewater, 42 $44^{\prime}$ 'S, 147¹3'E (TMAG J3013, J1626); 1 female, Brighton, Brighton Army Camp, 42॰42'S, $147^{\circ} 15^{\prime} \mathrm{E}$ (TMAG J2133); 1 female, Campania, 42³9'S, $147^{\circ} 25^{\prime} \mathrm{E}$ (TMAG J3012); 1 female, Claremont, $42^{\circ} 47^{\prime} \mathrm{S}, 147^{\circ} 15^{\prime} \mathrm{E}$ (TMAG J746); 1 male, Cressy, $41^{\circ} 40^{\prime} \mathrm{S}, 147^{\circ} 04^{\prime} \mathrm{E}$ (QVMAG 13:39861); 1 female, Dodges Ferry, 42 $51^{\circ} \mathrm{S}, 1^{\circ} 7^{\circ} 37^{\prime} \mathrm{E}$ (TMAG J1627);

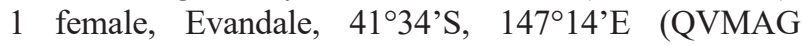
13:44937); 1 female, Flagstaff Gully, $42^{\circ} 51^{\prime} \mathrm{S}, 147^{\circ} 22^{\prime} \mathrm{E}$ (TMAG J2389); 3 males, Flat Rock Reserve, Chauncy Vale, 42 ${ }^{\circ} 37^{\prime} \mathrm{S}, 147^{\circ} 15^{\prime} \mathrm{E}$ (TMAG J4172, J4180, J4306); 2 males, Gagebrook, 42 ${ }^{\circ} 45^{\prime} \mathrm{S}, 147^{\circ} 16^{\prime} \mathrm{E}$ (TMAG J1761); 1 female, Geilston Bay, 42 $50^{\circ}$ S, $147^{\circ} 21^{\prime} \mathrm{E}$ (TMAG J632); 1 female, Hobart, 42 52 'S, $147^{\circ} 19^{\prime}$ E (TMAG J697); 1 female, same locality (TMAG J863); 1 female, same locality (NMV K8256); 1 female, same locality (TMAG J444); 1 female, Howrah, 42 $52^{\prime} \mathrm{S}, 147^{\circ} 23^{\prime} \mathrm{E}$ (TMAG J865); 1 male, Kempton, $42^{\circ} 31^{\prime} \mathrm{S}, 147^{\circ} 12^{\prime} \mathrm{E}$ (TMAG J1393); 1

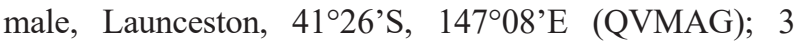
males, same locality (QVMAG); 1 male, same locality (QVMAG); 1 female, same locality (AM KS10412); 1 female, same locality (QVMAG 1957.13.42); 1 male, same locality (QVMAG); 1 female, same locality (QVMAG); 1 female, Launceston, 11 Pitt Ave, 41 $26^{\prime} \mathrm{S}, 147^{\circ} 08^{\prime} \mathrm{E}$ (QVMAG); 1 female, Launceston, 125 High St, 41 ${ }^{\circ} 26^{\prime} \mathrm{S}$, $147^{\circ} 08^{\prime} \mathrm{E}$ (QVMAG); 1 female, Launceston, 14 Dandenong Road, 4126'S, $147^{\circ} 08^{\prime} \mathrm{E}$ (QVMAG 13:44918); 1 female, 1 juv., Launceston, 3 Galvan St, $41^{\circ} 26^{\prime}$ S, $147^{\circ} 08^{\prime} \mathrm{E}$ (QVMAG); 1 female, Launceston, 3 Gorge Rd, 41 ${ }^{\circ} 26^{\prime}$ S, $147^{\circ} 08^{\prime} \mathrm{E}$ (QVMAG); 1 female, Launceston, 4 Eurella St, $41^{\circ} 26^{\prime} \mathrm{S}, 147^{\circ} 08^{\prime} \mathrm{E}$ (QVMAG); 1 female, Launceston, 42a Quarantine Road, $41^{\circ} 26^{\prime} \mathrm{S}, 147^{\circ} 08^{\prime} \mathrm{E}$ (QVMAG); 1 female, Launceston, High St, $41^{\circ} 26^{\prime} \mathrm{S}, 147^{\circ} 08^{\prime} \mathrm{E}$ (QVMAG

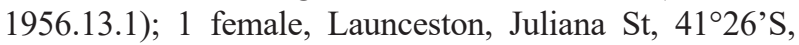
$147^{\circ} 08^{\prime} \mathrm{E}$ (QVMAG ); 1 male, Launceston, Peel St, 4126'S, $147^{\circ} 08^{\prime} \mathrm{E}$ (QVMAG 13:43529); 1 female, Launceston, St Mary St, 41 ${ }^{\circ} 26^{\prime} \mathrm{S}, 14^{\circ} 08^{\prime} \mathrm{E}$ (QVMAG 13:44931); 1 female, Launceston, Thistle St, $41^{\circ} 26^{\prime}$ S, $147^{\circ} 08^{\prime} \mathrm{E}$ (QVMAG ); 1 male, Launceston, Velodrome, 4126'S, $147^{\circ} 08^{\prime} \mathrm{E}$ (QVMAG 13:44939); 2 females, Lutana, 42 50'S, $147^{\circ} 18^{\prime} \mathrm{E}$ (TMAG J665, J3129); 2 males, Midway Point, $42^{\circ} 48^{\prime} \mathrm{S}, 147^{\circ} 31^{\prime} \mathrm{E}$ (TMAG J745, J1118); 2 females, Moonah, 42 50 'S, $147^{\circ} 17^{\prime} \mathrm{E}$ (TMAG J1117, J1293); 1 female, northern Tasmania (no exact locality) (QVMAG 13:43026); 1 female, Orford, near Orford rivu- 

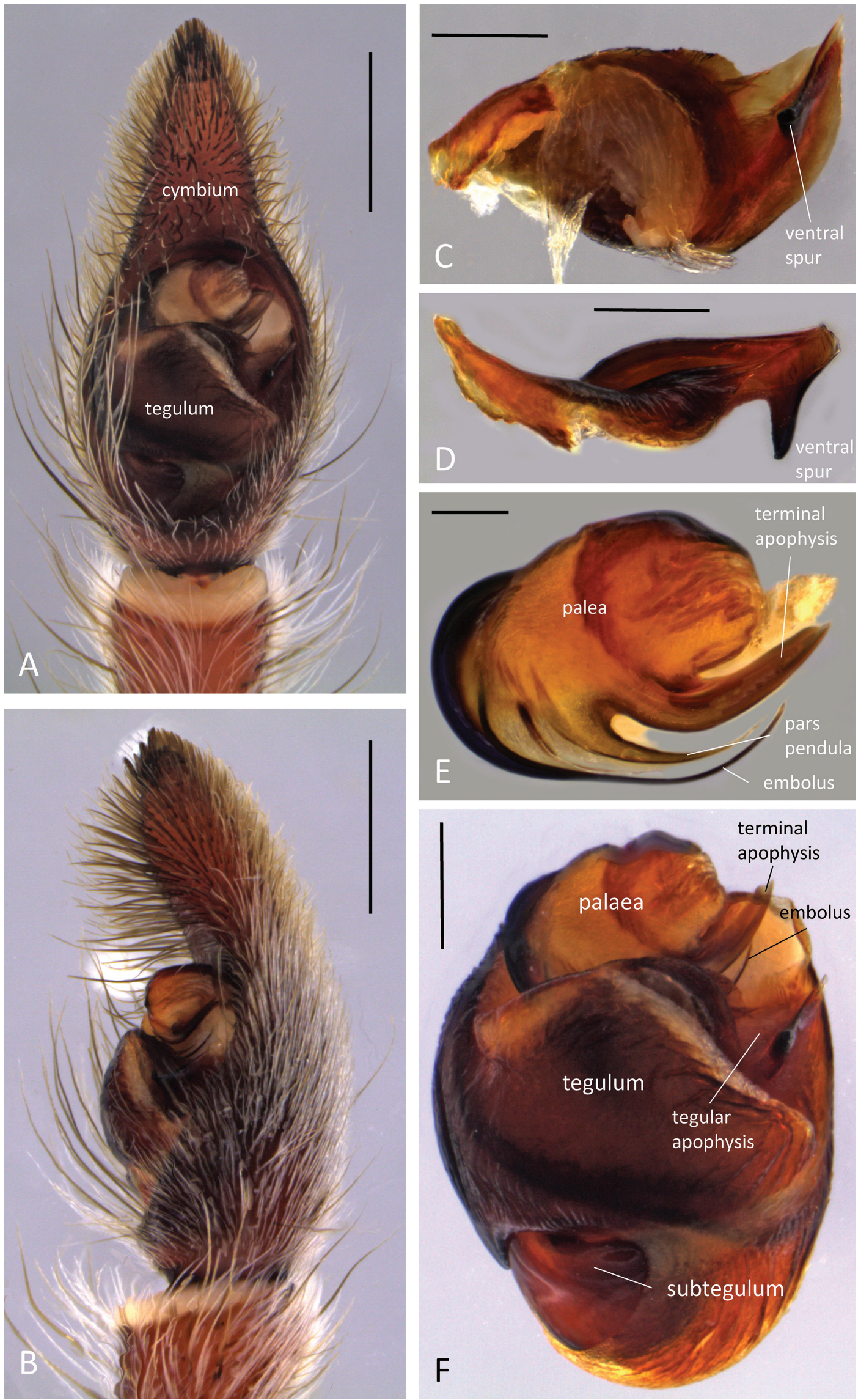

Figure 3. Portacosa cinerea gen. n. and sp. n., male holotype (WAM T68032), pedipalp. A, B, pedipalp, ventral (A) and retrolateral (B) view. C, D, tegular apophysis, ventral (C) and apical (D) view. E, palea, ventral view. F, pedipalp bulb (dissected from cymbium), ventral view. Scale bar: A, B $-0.75 ; \mathbf{C}, \mathbf{D}-0.25 ; \mathbf{E}-0.11, \mathbf{F}-0.34$. 
let, 42 $34^{\prime}$ 'S, $147^{\circ} 52^{\prime} \mathrm{E}$ (TMAG J892); 1 female, Porter Hill, 42 $55^{\prime} 24^{\prime}$ 'S, $147^{\circ} 21$ '18'E (TMAG J4126); 1 female, Queens Domain, Hobart, 4252'S, 147 $19^{\prime} \mathrm{E}$ (AM KS30552); 1 female, Richmond, 42 $44^{\prime}$ 'S, $147^{\circ} 26^{\prime} \mathrm{E}$ (TMAG J2695); 1 female, Risdon Brook Reservoir, 4248'S, 147¹9'E (TMAG J2694); 1 male, Rosetta, Glenorchy, 42 $49^{\circ} \mathrm{S}, 147^{\circ} 15^{\prime} \mathrm{E}$ (TMAG J862); 1 male, South Hobart, 42 53 'S, $147^{\circ} 18^{\prime} \mathrm{E}$ (TMAG J2844); 2 females, same locality (TMAG J474, J1165); 1 male, St Leonards area, Launceston, $41^{\circ} 27^{\prime} \mathrm{S}, 147^{\circ} 12^{\prime} \mathrm{E}$ (QVMAG ); 2 females, Trevallyn, $41^{\circ} 26^{\prime} \mathrm{S}, 147^{\circ} 07^{\prime} \mathrm{E}$ (QVMAG 13:44912, 13:44932); 1 female, same locality (QVMAG); 1 female, Tunbridge, $42^{\circ} 08^{\prime} \mathrm{S}, 1^{\circ} 7^{\circ} 25^{\prime} \mathrm{E}$ (QVMAG 13:43226); 1 female, Waverley, 3 Fryet Street, $41^{\circ} 26^{\prime}$ 'S, $147^{\circ} 11^{\prime} \mathrm{E}$ (QVMAG); 1 female, West Launceston, $41^{\circ} 27^{\prime} \mathrm{S}, 147^{\circ} 07^{\prime} \mathrm{E}$ (QVMAG 13:44938). Victoria: 1 male, $6 \mathrm{~km}$ N of Baddaginnie, Basin Road, 36 32'24'S, 14550'01'E (WAM T56463); 1 female, Belmont, $38^{\circ} 10^{\prime}$ 'S, $144^{\circ} 20^{\prime} \mathrm{E}$ (NMV K8187); 1 male, Birthday Tank,

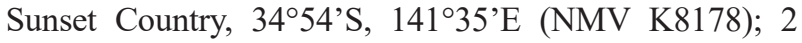
males, Gypsum, 3516'S, $142^{\circ} 23^{\prime} \mathrm{E}$ (NMV K8192); 1 female, Kooloonong, $34^{\circ} 53^{\prime} \mathrm{S}, 1^{\circ} 3^{\circ} 08^{\prime} \mathrm{E}$ (NMV K8184); 1 male, 1 juv., Merbein, $34^{\circ} 10^{\prime} \mathrm{S}, 142^{\circ} 04^{\prime} \mathrm{E}$ (AM KS36926); 1 male, Reservoir, 37²3'S, 14500'E (NMV K8193); 1 female, Runnymede Flora Reserve, $600 \mathrm{~m}$ along Two Chain Road from Elmore/Clonbinane Road, 36 35'20"S, 14443'54'E (WAM T56072); 1 female, Specimen Hill Bushland Reserve, Bendigo Regional Park, 3646'29'S, 144⒈'08'E (WAM T56062); 1 male, Werribee, Dept of Agriculture, Animal Research Institute, 3759'S, 144³3'E (NMV K8140).

Diagnosis. Only species known in genus (see generic Diagnosis above).

Description. Male (based on holotype, WAM T68032). Carapace. Brown to dark reddish-brown; indistinct dark radial pattern; centrally somewhat lighter (Fig. 2A); grey and few black setae, denser in eye region, in particular medially of PLE (Fig. 2A). Eyes. Row of anterior eyes slightly procurved and narrower than row of PME. Sternum. Light brown; few brown setae of variable length (Fig. 2B). Labium. Dark brown; front end truncated and white (Fig. 2B). Chelicerae. Very dark reddish-brown; covered frontally with grey dense setae. Cymbium tip with ca. 20 macrosetae (Fig $3 \mathrm{~A}, \mathrm{~B})$; tegular apophysis with ventral spur that connects to apical point with sharp edge (Fig. 3C, D); embolus and pars pendula slender, terminal apophysis very broad (Fig. 3E). Abdomen. Laterally olive-brown, dorsally lighter and with dark heart mark, covered in dense grey setae (Fig. 2A). Venter uniformly yellow-brown, covered with dense white and few longer brown setae (Fig. 2B). Spinnerets light brown. Legs. Leg formula IV $>$ I > II > III; uniformly brown; dense scopulate setae ventrally on tarsi and metatarsi of leg I and II. Spination of leg I: femur: 3 dorsal, 2 retrolateral, 2 prolateral; patella: 1 prolateral, 1 retrolateral; tibia: 3 ventral pairs, 2 prolateral, 2 retrolateral; metatarsus: 3 ventral pairs, 3 prolateral, 3 retrolateral, 1 apicoventral.
Female (based on paratype, WAM T53550). Carapace. Brown, with indistinct darker radial pattern present; uniformly dense grey setae (Fig. 2C). Eyes. Row of anterior eyes slightly procurved and narrower than row of PME. Labium, sternum, chelicerae. As male (paratype female with unidentified dark colouration on posterior sternum and right coxae II and III - potentially burn?) (Fig. 2D). Abdomen. Uniformly light brown, covered with grey setae (Fig. 2C). Venter and spinnerets as male (Fig. 2D). Epigyne. Median septum inverted T-shaped that widens anteriorly and with edges continuously forming distinct anterior hoods, atrium deep (Fig. 4A, C); spermathecal heads oval, wider than spermathecal stalks, spermathecal stalks S-shaped bent (Fig. 4B). Legs. Leg formula IV > I > II > III; uniformly brown; dense scopulate setae on tarsi and metatarsi of all legs and apical half of tibiae I. Spination of leg I: Femur: 2 dorsal, 3 retrolateral, 2 apicoprolateral; patella: 1 prolateral; tibia: 3 ventral pairs, 1 prolateral; metatarsus: 3 ventral pairs, 1 apicoventral.

Measurements. Male holotype, WAM T68032 (female paratype, WAM T53550): TL 13.9 (20.0), CL 8.2 (10.1), CW 5.5 (7.9). Eyes: AME 0.41 (0.49), ALE 0.22 (0.32), PME 0.79 (1.11), PLE 0.68 (1.03). Row of eyes: ALE 1.35 (1.89), PME 1.62 (2.32), PLE 2.01 (2.97). Sternum (length/width) 3.6/2.8 (3.7/3.1). Labium (length/width) 1.00/0.91 (1.4/1.3). AL 5.8 (10.3), AW 4.2 (8.1). Legs: Lengths of segments (femur + patella/tibia + metatarsus + tarsus $=$ total length): Pedipalp $3.6+3.1+-+2.6=9.3, \mathrm{I}$ $7.1+8.0+6.6+3.6=25.4$, II $6.6+8.0+6.5+3.6=24.7$, III $6.5+7.1+6.5+3.6=23.6$, IV $7.7+8.8+8.3+3.6$ $=28.4$ (Pedipalp $4.1+3.9+-+2.7=10.7$, I $7.00+8.4$ $+5.4+2.8=23.6$, II $6.8+8.1+5.4+3.0=23.3$, III 6.4 $+7.7+5.8+3.2=23.1$, IV $7.6+9.3+7.7+3.2=27.8)$.

Variation. Males (females) TL 9.5-14.5 (TL 15.0-25.0). Live specimens are generally uniformly grey in colour based on their dense pubescence, in particular if freshly moulted. Females have two small dark spots on the anterior half of their abdomen (Fig. 1C, D). Older specimens, in particular males, may have some discernible patterning due to the loss of setae over time (Fig. 1A, B).

Etymology. The specific epithet is a Latin adjective (cinereus - grey), referring to the colour and common name of the species, Grey Wolf Spider.

Distribution. South-eastern mainland Australia from south-east Queensland into South Australia, and Tasmania (Fig. 5)

Life history and habitat preferences. Mature females have been found throughout the year with the exception of August. Numbers gradually increase peaking in January, to decrease gradually to July. Females with eggsac or spiderlings have generally been found from November to February, with a single female with eggsac in collections recorded in April. Mature males have been found from October to May, with two distinct peaks of activity in November/December and March. These more extensive phenology data based on specimens examined for this study extend the seasonal activity for the species as previously reported for Tasmania, where females con- 

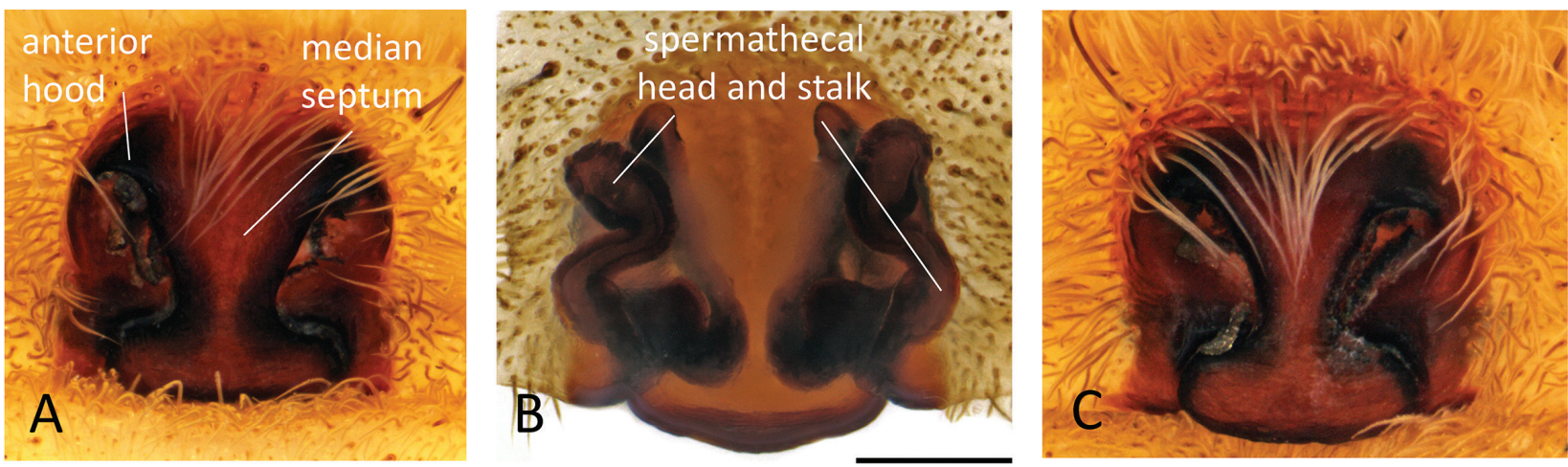

Figure 4. Portacosa cinerea gen. n. and sp. n., female paratype (WAM T53550) and non-type female from Runnymede Flora Reserve, Victoria (WAM T56072). A, B, female paratype, epigyne in ventral (A) and dorsal (B) view, C, non-type female, epigyne, ventral view. Scale bar: A, B, C -0.45 .

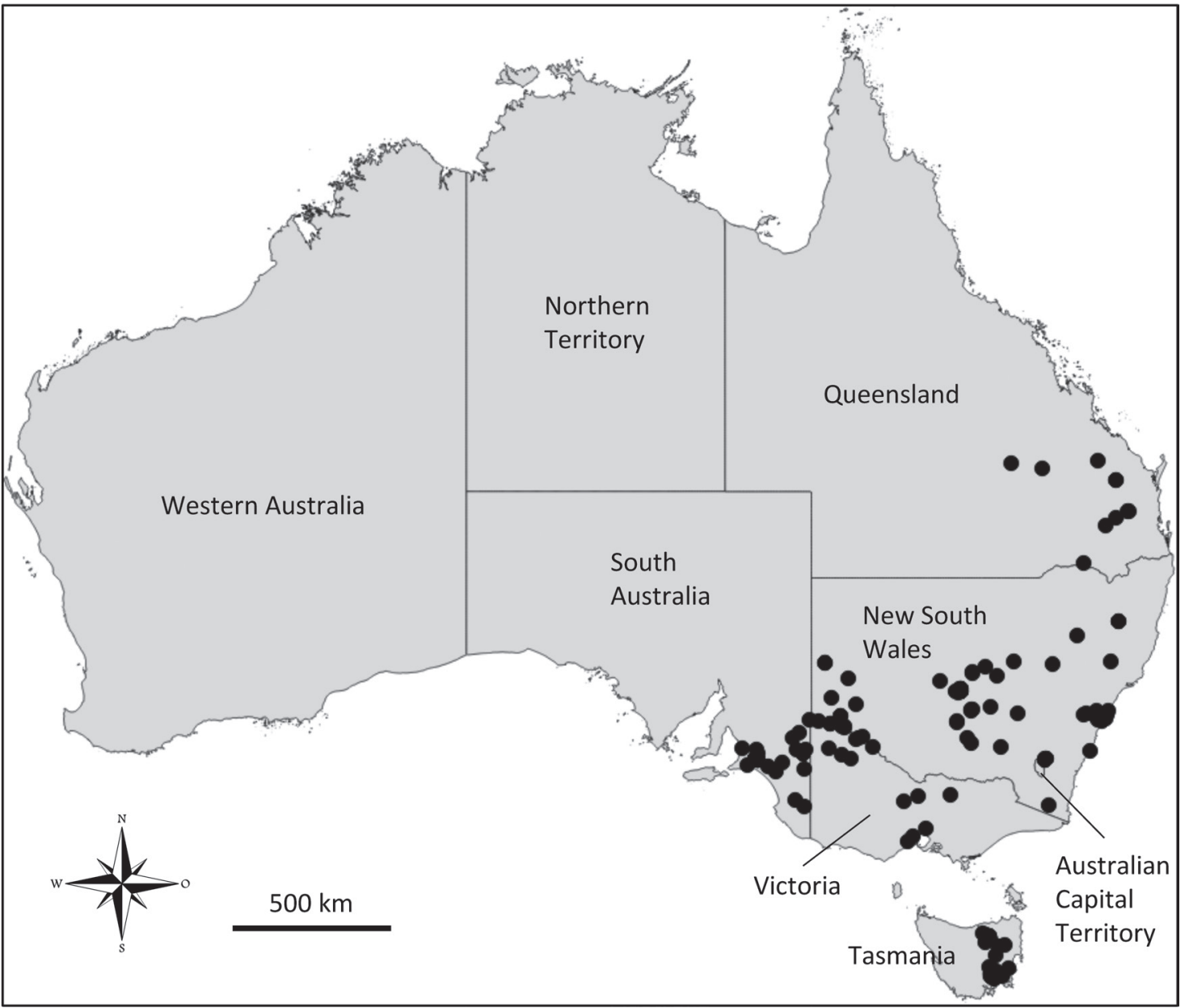

Figure 5. Distribution records of Portacosa cinerea gen. n. and sp. n. in Australia.

struct an eggsac in early summer and adults are found from January to April (Hickman 1967).

The Grey Wolf Spider has been reported from a number of generally open habitats, including (based on label data found with specimens): spinifex grassland, semi-arid grassland, River Red Gum (Eucalyptus camaldulensis) woodlands (including river banks), mallee (Acacia) woodlands, and Box-Ironbark (or Black Box) wood- lands. The species also occurs in cultural landscapes where it was found on road verges and in gardens or backyards, with males sometimes straying into houses. Immature and mature female spiders live in a straight burrow of approximately $20 \mathrm{~cm}$ depth, often constructed in heavily compacted soils, which they close with a plug-like trapdoor (Hickman 1967; VWF, personal observation) (Fig. 1G, H). 


\section{Acknowledgements}

I am indebted to the curatorial staff (some now retired) of the following institutions for either loans of specimens or their hospitality during respective visits: Graham Milledge, Helen Smith (AM); Bruce Halliday, Barry Richardson (ANIC); Christine Rollard (MNHP); Peter Lillywhite, Ken Walker, Catriona McPhee, Richard Marchant (NMV); Robert Raven, Owen Seeman, Barbara Baehr (QM); Lisa Boutin (QVMAG); David Hirst (formerly SAM); Liz Taylor (TMAG), Mark Harvey, Julianne Waldock (WAM). Shar Ramamurthy provided accommodation in Mildura, where the photographs of Fig. 1A, F, G were taken. Joseph Koh provided accommodation during a visit to Canberra where some specimens of this study were collected (Fig. 1F, H). I am grateful to Barbara Baehr (Brisbane) and Helen Smith (Sydney) for constructive suggestions that improved the manuscript.

\section{References}

Dondale CD (1986) The subfamilies of wolf spiders (Araneae: Lycosidae). Actas X Congreso Internacional de Aracnología, Jaca, España $327-332$.

Framenau VW (2006a) Knoelle, a new monotypic wolf spider genus from Australia (Araneae: Lycosidae). Zootaxa 1281: 55-67.

Framenau VW (2006b) Mainosa, a new genus for the Australian 'shuttlecock wolf spider' (Araneae, Lycosidae). Journal of Arachnology 34: 206-213. https://www.doi.org/10.1636/H04-90.1

Framenau VW (2015) Review of the Australian wolf spider genus Venator (Araneae, Lycosidae). Zootaxa 4013: 541-555. https://doi. org/10.11646/zootaxa.4013.4.5

Framenau VW, Baehr BC (2007) Revision of the Australian wolf spider genus Dingosa Roewer, 1955 (Araneae, Lycosidae, Lycosinae). Journal of Natural History 41: 1603-1629. https://www.doi. org/10.1080/00222930701475717

Framenau VW, Baehr BC (2016) Revision of the Australian Union-Jack wolf spiders, genus Tasmanicosa (Araneae, Lycosidae, Lycosinae). Zootaxa 4213: 1-82. https://doi.org/10.11646/zootaxa.4213.1.1

Framenau VW, Leung AE (2013) Costacosa, a new genus of wolf spider (Araneae, Lycosidae) from coastal north-west Western Australia. Records of the Western Australian Museum, Supplement 83: 173-184. https://www.doi.org/10.18195/issn.0313-122x.83.2013.173-184
Framenau VW, Main BY, Waldock JM, Harvey MS (2009) Tapetosa, a new monotypic wolf spider genus from Western Australia (Araneae: Lycosidae: Lycosinae). Records of the Western Australian Museum 25: 309314. https://www.doi.org/10.18195/issn.0312-3162.25(3).2009.309-314

Framenau VW, Vink CJ (2001) Revision of the wolf spider genus Venatrix Roewer (Araneae: Lycosidae). Invertebrate Taxonomy 15: 927-970. https://www.doi.org/10.1071/IT01008

Framenau VW, Yoo J-S (2006) Systematics of the new Australian wolf spider genus Tuberculosa (Araneae, Lycosidae). Invertebrate Systematics 20: 185-202. https://doi.org/10.1071/IS05036

Hickman VV (1967) Some common Spiders of Tasmania. Tasmanian Museum and Art Gallery, Hobart, 112 pp.

Hogg HR (1900) A contribution to our knowledge of the spiders of Victoria: including some new species and genera. Proceedings of the Royal Society of Victoria (N.S.) 13: 68-123.

Langlands PR, Framenau VW (2010) Systematic revision of Hoggicosa Roewer, 1960, the Australian 'bicolor' group of wolf spiders (Araneae: Lycosidae). Zoological Journal of the Linnean Society 158 : 83-123. https://www.doi.org/10.1111/j.1096-3642.2009.00545.x

Mello-Leitão CF de (1941) Las arañas de la provincia de Santa Fe colectadas por el Profesor Birabén. Revista del Museo de La Plata (N.S., Zool.) 2: 199-225.

Murphy NP, Framenau VW, Donnellan SC, Harvey MS, Park Y-C, Austin AD (2006) Phylogenetic reconstruction of the wolf spiders (Araneae: Lycosidae) using sequences from the 12S rRNA, 28S rRNA, and NADH1 genes: implications for classification, biogeography, and the evolution of web building behaviour. Molecular Phylogenetics and Evolution 38: 583-602. https://doi.org/10.1016/j.ympev.2005.09.004

Roewer CF (1955a) Die Araneen der Österreichischen Iran-Expedition 1949/50. Sitzungsberichte der Österreichischen Akademie der Wissenschaften. Mathematisch-naturwissenschaftliche Klasse 164: 751-782.

Roewer CF (1955b) Katalog der Araneae von 1758 bis 1940, bzw 1954. Vol. 2. Institut Royal de Sciences Naturelles de Belgique, Bruxelles, $1751 \mathrm{pp}$.

Roewer CF (1959) Araneae Lycosaeformia II. (Lycosidae). In: Institute des Parcs Nationaux du Congo et du Rwanda (Ed.) Exploration du Parc National de l'Upemba - Mission G. F. de Witte, Vol. 55, 1-518.

Roewer CF (1960d) Araneae Lycosaeformia II (Lycosidae) (Fortsetzung und Schluss). In: Institute des Parcs Nationaux du Congo et du Rwanda (Ed.) Exploration du Parc National de l'Upemba, Mission G. F. de Witte 55, 519-1040.

Simon E (1898) Descriptions d'arachnides nouveaux des familles des Agelenidae, Pisauridae, Lycosidae et Oxyopidae. Annales de la Société Entomologique de Belgique 42: 5-34.

Sundevall CJ (1833) Conspectus Arachnidum. Londini Gothorum, 39 pp. 